\title{
Conductance oscillations in mesoscopic rings: Microscopic versus global phase
}

\author{
R. Häussler* \\ Physikalisches Institut, Universität Karlsruhe, D-76128 Karlsruhe, Germany \\ E. Scheer ${ }^{\dagger}$ \\ Fachbereich Physik, Universität Konstanz, D-78457 Konstanz, Germany
}

H. B. Weber

Forschungszentrum Karlsruhe, Institut für Nanotechnologie, D-76021 Karlsruhe, Germany

H. v. Löhneysen

Physikalisches Institut, Universität Karlsruhe, D-76128 Karlsruhe, Germany

and Forschungszentrum Karlsruhe, Institut für Festkörperphysik, D-76021 Karlsruhe, Germany

(Received 31 March 2001; published 2 August 2001)

\begin{abstract}
The phase of Aharonov-Bohm oscillations in mesoscopic diffusive metal rings in the presence of a magnetic field, can be modulated by the application of a dc-bias current $I_{\mathrm{dc}}$. We address the question of how a variation of $I_{\mathrm{dc}}$ and hence of the microscopic phases of the electronic wave functions results in the observable "global" phase of the conductance oscillations. Whereas the first one can be varied continuously, the latter has to be quantized for a ring in a two-wire configuration by virtue of the Onsager symmetry relations. We observe a correlation between a phase flip by $\pm \pi$ and the amplitude of the oscillations.
\end{abstract}

PACS number(s): 73.23.-b, 73.50.-h, 85.35.Ds

\section{INTRODUCTION}

The fundamental concept of time-reversal symmetry has important implications on the symmetry of transport properties. Onsager succeeded in deriving general reciprocity relations from the principle of microscopic reversibility. ${ }^{1}$ The application of the Onsager relations (OR) to the electrical conductance as a macroscopic quantity, was discussed by Casimir. ${ }^{2}$ When quantum interference effects are negligible, they imply a symmetry condition for the magnetoconductance (MC) with respect to sign reversal of the field, i.e., $G(B)=G(-B)$. In mesoscopic devices, however, the symmetry of the MC upon magnetic-field reversal depends on the sample geometry. ${ }^{3}$ It is necessary to distinguish between mesoscopic two-wire and four-wire configurations. In a twowire configuration, the current and voltage leads branch outside the phase-coherent region of the electrons and the conductance $G$ is indeed symmetric with respect to the magnetic field $B$, as in macroscopic samples. ${ }^{3-5}$ This simple symmetry relation does not hold for a four-wire configuration with a bifurcation of the voltage and current leads within the phasecoherent region. In this geometry, the symmetry is only observed when simultaneously reversing voltage and current probes: $G_{U, I}(B)=G_{I, U}(-B)$. The latter has been confirmed experimentally by Benoit et al. ${ }^{6}$ by studying conductance oscillations of mesoscopic rings.

In mesoscopic rings exposed to a perpendicular magnetic field $B$, the interference of partial waves of electrons propagating phase coherently in opposite directions, leads to oscillations of $G$ with a fundamental period $B_{p e r}=\phi_{0} / A$, where $\phi_{0}=h / e$ is the flux quantum and $A$ the enclosed area of the ring. ${ }^{7}$ In rings with finite width of the arms, the interference of waves within one arm of the ring results in a modulation of the amplitude and the phase of the oscillations: ${ }^{8}$

$$
\Delta G=g(B, E) \cos \left[2 \pi \frac{B A}{\phi_{0}}+\varphi(B, E)\right] .
$$

The oscillations of $\Delta G$ with respect to $B$ are usually termed the Aharonov-Bohm (AB) effect although strictly speaking the $A B$ effect, in its original meaning, refers to a phase shift of electron waves by a vector potential only, i.e., with vanishing magnetic field along the electrons' trajectories. ${ }^{9}$ The amplitude $g$ and the phase $\varphi$ are sample specific as they depend on the microscopic arrangement of the scattering centers in the ring. Both quantities are random functions of the magnetic field $B$ and the energy $E$ of the electrons. The typical scales in $B$ and $E$ for a variation of $g$ and $\varphi$, are the correlation field $B_{c, A B} \approx \phi_{0} / 2 w L$ and the Thouless energy $E_{c, A B} \sim h D / L^{0.7} L_{\varphi}^{1.3}$, where $w$ is the width of the ring arms, $L$ the half-perimeter of the ring, $L_{\varphi}$ is the phase-coherence length, and $D$ is the diffusion constant. ${ }^{10-12}$

Hence, for a ring in a two-wire configuration, the symmetry condition does not allow arbitrary values of the phase of the $\mathrm{AB}$ oscillations. On the other hand, in a naive picture, this phase is determined by the arbitrary (but fixed for a given field) phase difference between electron partial waves traveling through the two arms, which in turn depends on the phase an electron accumulates at the scattering centers in the metal ring during its diffusive motion. Consequently, a continuous phase shift of the $\mathrm{AB}$ oscillations would be expected.

In our experiment, we address the question of how the quantization condition of the macroscopically observable phase of the conductance oscillations is fulfilled when the microscopic electronic phase is varied continuously. Most of the previous experiments on OR in diffusive metal rings with continuous voltage drop along the ring arms, ${ }^{6,13}$ investigated only "snap shots" of a given distribution of microscopic phases, since they compared the behavior of microscopically 
TABLE I. Parameters of four representative samples, on which the data presented in this paper have been taken; configuration refers to mesoscopic two-wire or four-wire configuration. The $\star$ for sample No. 1, indicates that the bifurcation of the current and voltage leads into four wires was not directly at the ring, but at a distance of $500 \mathrm{~nm}$ from the ring. $\phi$ denotes the mean diameter of the rings, $w$ the width of the ring arms, $d$ the thickness of the deposited film, $R$ the total resistance of the sample including the ring and the leads, $R_{\text {ring }}$ is the resistance of the ring determined by subtracting from $R$ the calculated contributions of the leads assuming a constant resistivity $\rho$ and taking into account the geometry (measured from electron micrographs). The elastic mean-free-path $l$ has been determined from the residual resistance ratio RRR $=R(300 \mathrm{~K}) / R(4.2 \mathrm{~K}) \simeq 1+l / l_{e-p h} \quad$ (with the values for the electron-phonon scattering length $l_{e-p h}$ taken from literature) and independently by using the material-specific $\rho l$ products. The values determined by both methods agree within $20 \%$. The correlation field $B_{c, \mathrm{UCF}}$ has been determined from the UCF recorded in the magnetic field range from -8.5 to $8.5 \mathrm{~T}$ and the phase-coherence length $L_{\varphi}$ by $L_{\varphi}=\phi_{0} / w B_{c, \mathrm{UCF}}$.

\begin{tabular}{lrrrr}
\hline \hline Sample No. & 1 & 2 & 3 & 4 \\
\hline Metal & $\mathrm{Cu}$ & $\mathrm{Ag}$ & $\mathrm{Cu}$ & $\mathrm{Cu}$ \\
Configuration & $\star$ & 2 & 2 & 4 \\
$\phi(\mathrm{nm})$ & 1000 & 980 & 980 & 975 \\
$w(\mathrm{~nm})$ & 60 & 80 & 80 & 60 \\
$d(\mathrm{~nm})$ & 20 & 25 & 15 & 15 \\
$R(\Omega)$ & 61.0 & 26.2 & 60.5 & 55.6 \\
$R_{\text {ring }}(\Omega)$ & 33 & 16 & 38 & 55.6 \\
$l(\mathrm{~nm})$ & 14 & 25 & 12 & 10 \\
$B_{c, \mathrm{UCF}}(\mathrm{mT})$ & 38 & 53 & 62 & 62 \\
$L_{\varphi}(\mathrm{nm})$ & 1750 & 940 & 800 & 1070 \\
\hline \hline
\end{tabular}

different samples. Here we tune the microscopic behavior of a given sample, i.e., with identical distribution of scattering centers. By the application of a dc-bias current $I_{\mathrm{dc}}$ we generate a nonequilibrium energy distribution of the electrons in the ring since no energy relaxation occurs in the phasecoherent region. The energy of the electrons contributing to the charge transport, and hence also the phase of the microscopic electronic wave function, can be modified by a variation of $I_{\mathrm{dc}}$. We analyze the reaction of the global phase of the $\mathrm{AB}$ oscillations to this continuous variation on the microscopic level. Technically this is performed by monitoring the cross correlation for MC traces at different $I_{\mathrm{dc}}$.

\section{EXPERIMENT}

We show here data taken on four different samples (see Table I). The samples were prepared by electron-beam lithography and thermal evaporation of $\mathrm{Cu}$ or $\mathrm{Ag}$ on a $\mathrm{Si}$ substrate at room temperature followed by a lift-off process. The differential resistance $d V / d I_{\text {ac }}$ was measured at $19 \mathrm{~Hz}$ (typically $I_{\mathrm{ac}}=100 \mathrm{nA}$ ) with a superimposed dc-bias current $I_{\mathrm{dc}}$ with the sample mounted inside the mixing chamber of a dilution refrigerator with a base temperature of $20 \mathrm{mK}$. The energy scale $E_{\mathrm{ac}}=e I_{\mathrm{ac}} R$ related to the ac current, was always
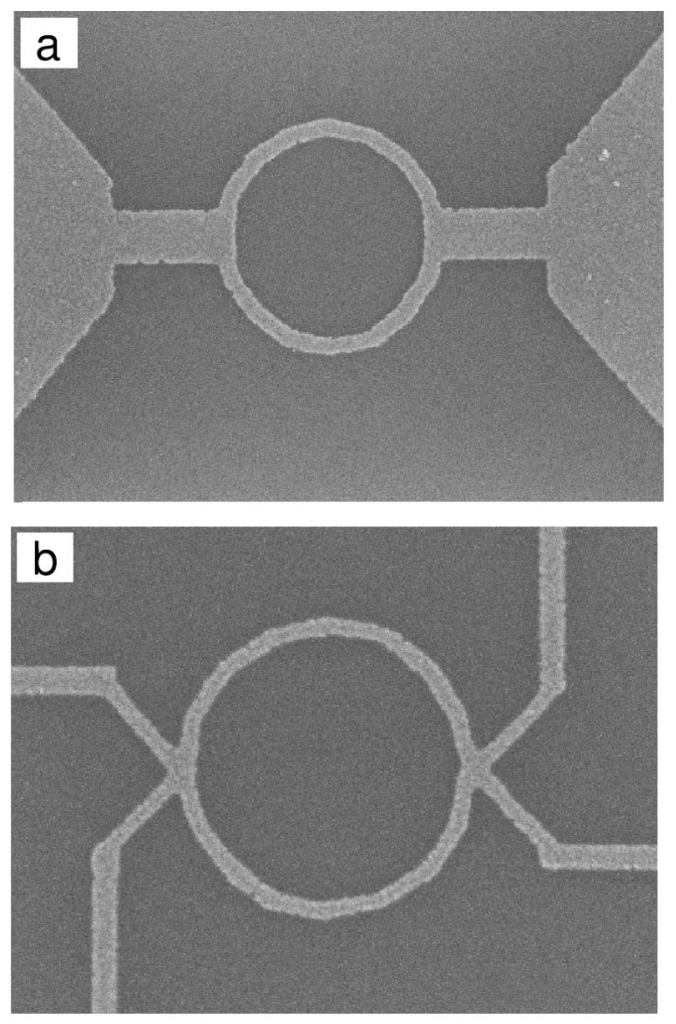

FIG. 1. Electron micrographs of (a) sample No. 3 in two-wire and (b) sample No. 4 in four-wire configuration. The rings have an average diameter $\phi$ of approximately $1 \mu \mathrm{m}$ and a linewidth of (a) $80 \mathrm{~nm}$ and (b) $60 \mathrm{~nm}$. For sample parameters, see Table I.

kept smaller than the thermal energy $E_{T}=k_{B} T$ and $E_{c}$. The refrigerator was equipped with a superconducting solenoid, allowing magnetic fields from -8.5 to $8.5 \mathrm{~T}$ with a resolution of $0.02 \mathrm{mT}$. Due to the hysteresis of the superconducting magnet, the absolute field values had to be corrected by an offset of $4.5 \mathrm{mT}$, which was reproducible within an error of $0.1 \mathrm{mT}$ when sweeping the field always with the same rate $\approx 1 \mathrm{mT} / \mathrm{min}$.

Figure 1 shows electron microscope photographs of two rings. In the two-wire configuration, the voltage and current leads branch at a distance of $4 \mu \mathrm{m}$ from the ring (not shown in the micrograph) which is more than the phase-coherence length $L_{\varphi}$ of $\approx 1 \mu \mathrm{m} . L_{\varphi}$ has been determined by analysis of the universal conductance fluctuations (UCF) measured simultaneously. ${ }^{14}$ In the four-wire configuration, the bifurcation is directly at the ring. For sample No. 1, the separation between current and voltage leads was at a distance of $500 \mathrm{~nm}$ from the ring. For this sample we observed a mixed behavior between two-wire and four-wire behavior (see below).

\section{RESULTS AND DISCUSSION}

Figure 2(a) displays the MC of sample No. 4 (in four-wire geometry) for $I_{\mathrm{dc}}=0$ measured at $T=20 \mathrm{mK}$ (without any filtering) for the field range of -6 to $6 \mathrm{~T}$, where we observe UCF. Obviously the MC is not exactly symmetric with respect to the magnetic field. The aperiodic, reproducible 

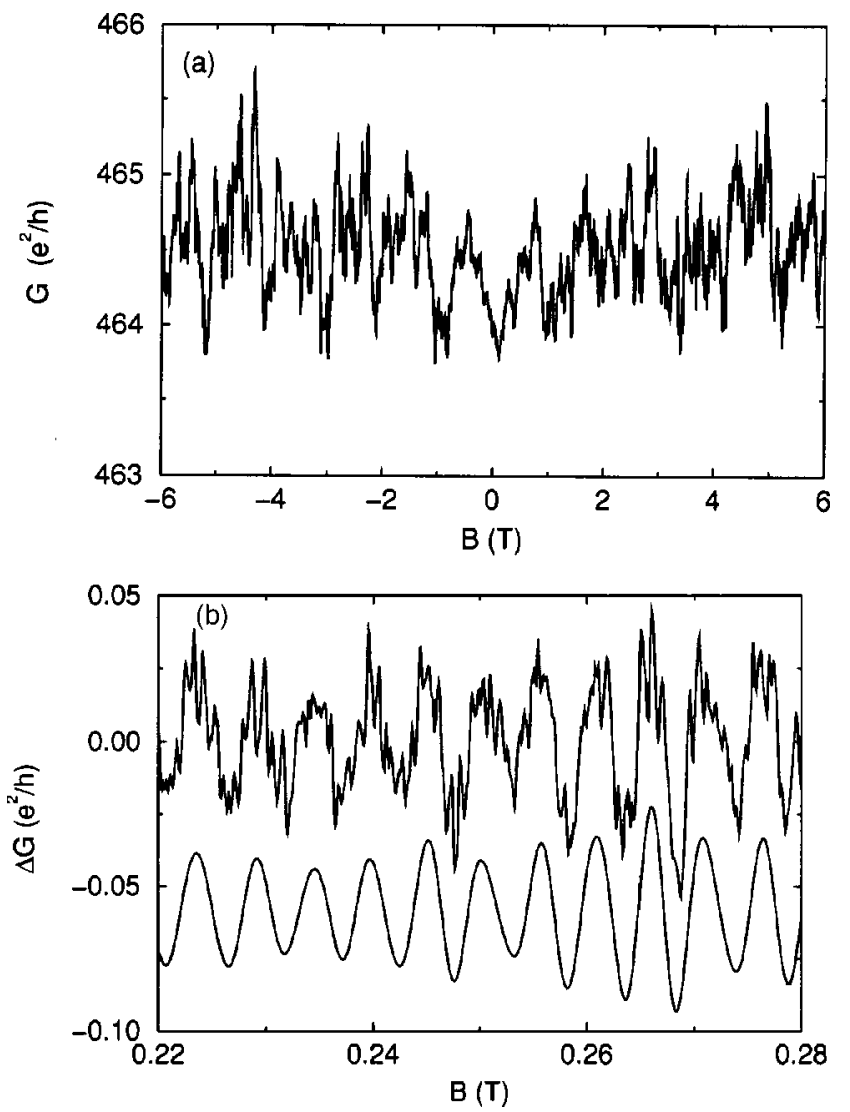

FIG. 2. (a) Conductance $G$ of sample No. 4 (four-wire configuration) vs magnetic field $B$ at current $I_{\mathrm{dc}}=0$ and a temperature $T$ $=20 \mathrm{mK}$. (b) Amplification of field range 0.22 to $0.28 \mathrm{~T}$ as measured (upper curve) and after Fourier filtering (lower curve). Only frequencies corresponding to a period range from 2 to $10 \mathrm{mT}$ were taken into account. The data are offset vertically for clarity and a linear background has been subtracted.

UCF were used for determining $B_{c, \mathrm{UCF}}$. In panel (b) we have plotted the unfiltered data (upper curve) and the Fourier filtered data for the smaller field range of $0.22 \mathrm{~T}<B<$ $0.28 \mathrm{~T}$, showing clearly the $\mathrm{AB}$ oscillations with a period of (5.2 \pm 0.5$) \mathrm{mT}$, in good agreement with the expected value $\Phi_{0} / A=5.3 \mathrm{mT}$. The filtering has been performed with a bandpass allowing only periods between 2 and $10 \mathrm{mT}$. Only for sample No. 1 with the largest $L_{\varphi}$, we observed contributions from the first harmonic of the $\mathrm{AB}$ oscillations with a period of $h / 2 e .{ }^{15}$ Consequently, we used for filtering those data, the wider period range of 2 to $20 \mathrm{mT}$ (see Fig. 8). The finite resolution of the period of the h/e oscillations is due to the finite width of the ring arms and, as explained below, to intrinsic period modulations enforced by time-reversal symmetry.

We observe equilibrium amplitudes $g(E=0, B)$ of the $h / e$ oscillations between $0.01 e^{2} / h$ for sample No. 3 and $0.06 e^{2} / h$ for sample No. 1. The expected saturation value for a fully phase-coherent sample would be $0.3 e^{2} / h$. When the length of the ring arms is larger than $L_{\varphi}, g(E=0, B)$ of the $\mathrm{AB}$ oscillations is exponentially suppressed by a factor $\left(2 L_{\varphi} / U\right) \exp \left[-U / L_{\varphi}\right]$, where $U$ is the circumference of the ring. ${ }^{16}$ With our sample parameters we estimate this

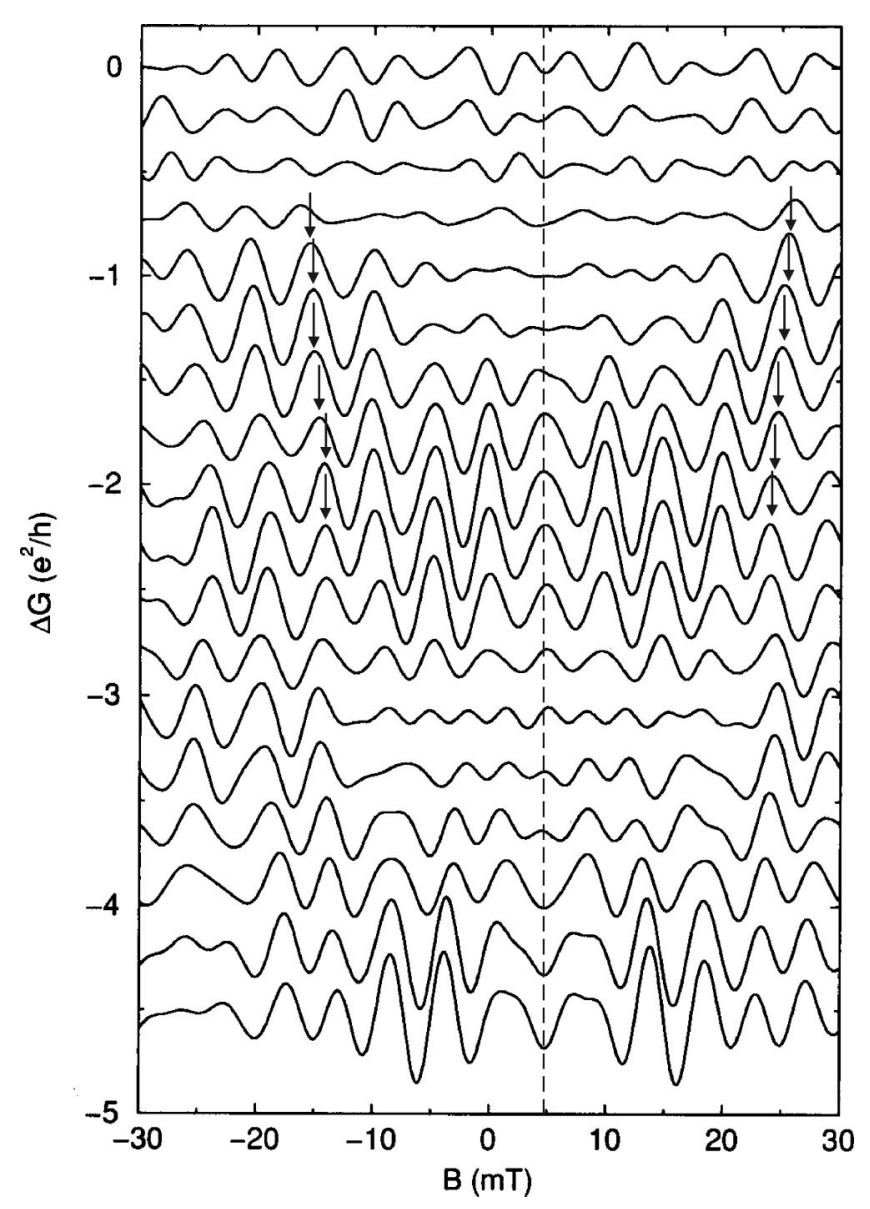

FIG. 3. Conductance oscillations $\Delta G$ of sample No. 2 vs magnetic field $B$ at currents $I_{\mathrm{dc}}=0,0.4,0.8, \ldots, 6.8 \mu \mathrm{A}$ (from top to bottom) and a temperature $T=90 \mathrm{mK}$. The data were digitally Fourier filtered. Only frequencies corresponding to a period range from 2 to $10 \mathrm{mT}$ were taken into account. The data are offset vertically for clarity. The effective zero field is at $B=4.5 \mathrm{mT}$.

reduction factor to be of the order of $\sim 0.1$ for sample No. 1 and $\sim 0.01 \pm 0.003$ for sample Nos. 2,3 , and 4 , in reasonable agreement with the experimental findings.

Figure 3 (Fig. 4) displays the evolution of the $\mathrm{AB}$ oscillations with applied dc-current for sample No. 2 (No. 4) in a two-wire (four-wire) configuration. The $\mathrm{AB}$ oscillations with a period of $5.2 \mathrm{mT}$, are clearly visible, and it is evident that their amplitude and phase vary with $I_{\mathrm{dc}}$ for both samples.

First we discuss the behavior of sample No. 2. At $I_{\mathrm{dc}}$ $=1.2 \mu \mathrm{A}$ (fourth curve from the top), the amplitude has a first minimum and the phase has flipped by $\pi$. Note that the offset of the field axis has not been subtracted. Therefore the $\mathrm{AB}$ oscillations are symmetric (within the measurement resolution) around the offset value $B=4.5 \mathrm{mT}$. Evidently, the precision with which the phase shifts can be determined, depends on the amplitude. For amplitudes larger than $\Delta G$ $\approx 0.02 e^{2} / h$, the error in the phase is smaller than $\pi / 4$. In a separate publication ${ }^{14}$ we showed that the average amplitude of the oscillations increases with increasing $I_{\mathrm{dc}}$. We used this effect, which has been studied in detail in a recent work by Terrier et $a l^{17}$ for the evaluation of the phase shift, to measure oscillations with larger amplitude at large $I_{\mathrm{dc}}$ and hence 


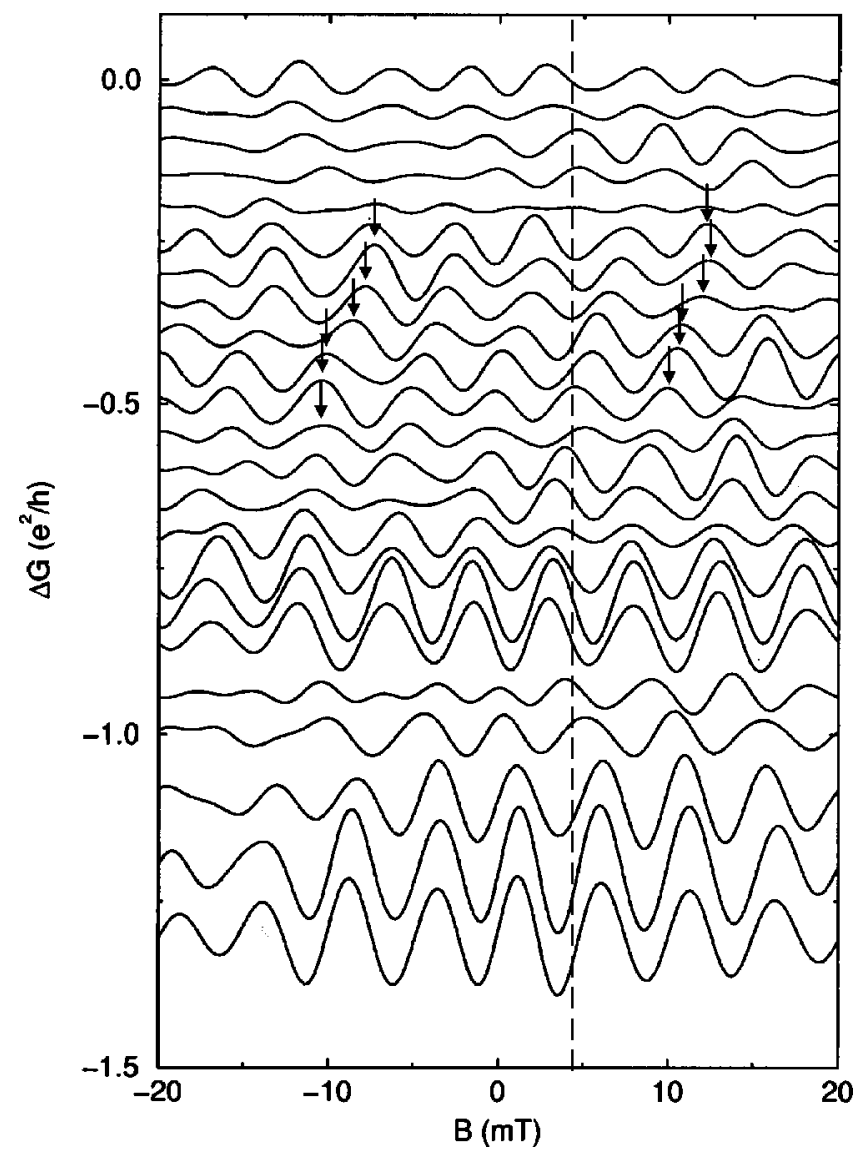

FIG. 4. Conductance oscillations $\Delta G$ of sample No. 4 vs magnetic field $B$ at currents $I_{\mathrm{dc}}=0,0.15,0.3, \ldots, 3.3 \mu \mathrm{A}$ (from top to bottom) and a temperature $T=90 \mathrm{mK}$. The data were digitally Fourier filtered. Only frequencies corresponding to a period range from 2 to $10 \mathrm{mT}$ were taken into account. The data are offset vertically for clarity. The effective zero field is at $B=4.5 \mathrm{mT}$.

to increase the signal-to-noise ratio.

The AB oscillations of sample No. 4 in four-wire configuration (Fig. 4) are also shifted with $I_{\mathrm{dc}}$, but they are not symmetric upon reversal of $B$, in agreement with theoretical prediction $^{3}$ and previous experimental observation. ${ }^{6}$

The average phase shift in the whole investigated magnetic-field range is analyzed quantitatively by calculating the cross-correlation function $(\mathrm{CCF})$ between the conductance oscillations measured at currents $I_{1}$ and $I_{2}$ :

$$
C\left(I_{1}, I_{2}, \Delta B\right)=\int \Delta G_{I_{1}}(B) \Delta G_{I_{2}}(B+\Delta B) d B
$$

The CCF of two periodic functions with the same period is again a periodic function. A shift of $\delta B$ between these functions manifests itself in a shift of the maxima of $C(\Delta B)$ by $\delta B$.

Figure 5(a) shows the CCF's of oscillations measured on sample No. 2. The CCF's are calculated for $\Delta G(B)$ taken at different $I_{2}=I_{\mathrm{dc}}$, each time with reference to $\Delta G(B)$ at $I_{1}$ $=0$. It can be seen that the oscillations are either in phase or shifted by $\delta B=B_{p e r} / 2$. For comparison, the evaluation of a
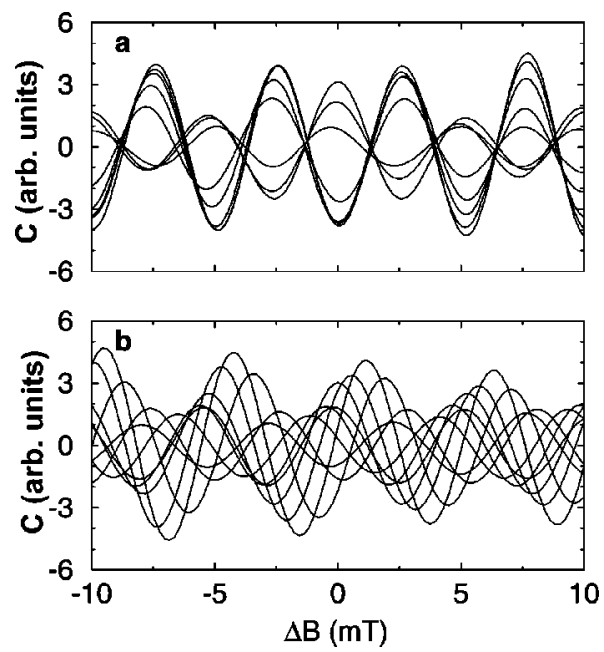

FIG. 5. (a) Cross-correlation functions $C\left(I_{1}, I_{2}, \Delta B\right)$ for $I_{1}=0$ and different $I_{2}=I_{\mathrm{dc}}$ vs $\Delta B$ for sample No. 2 in a two-wire configuration. The magnetoconductance is displayed in Fig. 2 and was evaluated in a magnetic-field range from -30 to $30 \mathrm{mT}$. (b) Crosscorrelation functions for sample No. 4 in four-wire configuration for comparison.

ring in a four-wire configuration, is displayed in Fig. 5(b). Here the shift $\delta B$ between the oscillations is arbitrary.

The phase shift of the oscillations $\delta \varphi$ is related to $\delta B$ by the relation $\delta \varphi=2 \pi \delta B / B_{\text {per }}$. The observed shift of the CCF's results in $\delta \varphi=0$ or $\delta \varphi=\pi$ for a two-wire configuration [cf. Fig. 6(a)] whereas $\delta \varphi$ is arbitrary for the four-wire configuration [cf. Fig. 6(b)]. This behavior demonstrates convincingly the expected symmetry relations as the $\mathrm{MC}$ of a
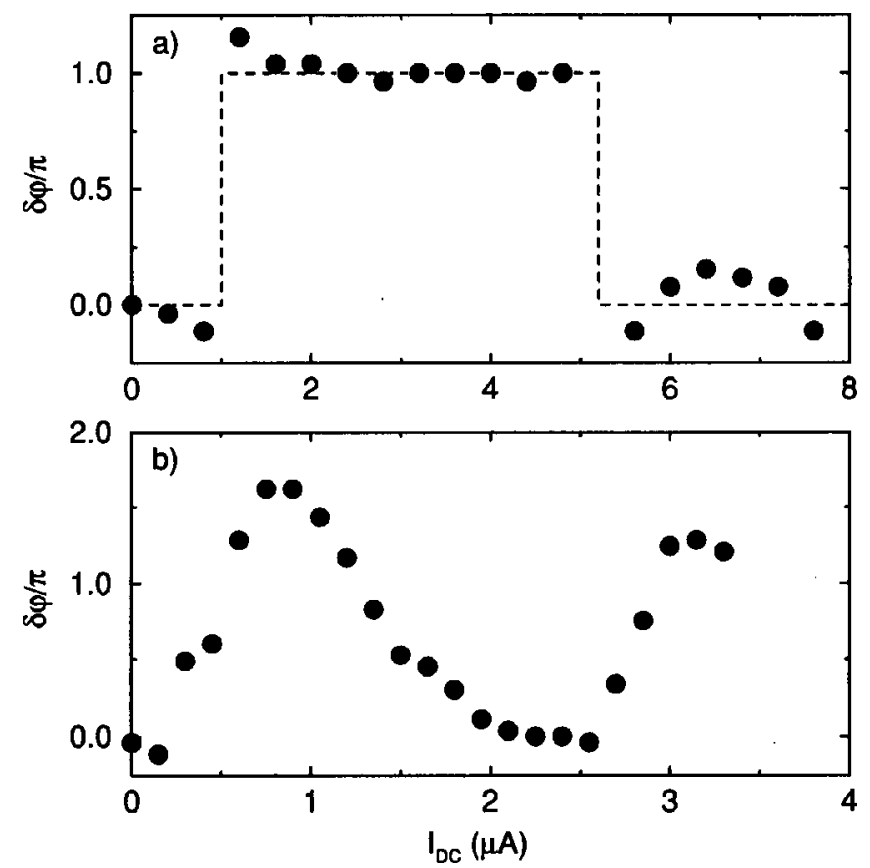

FIG. 6. Phase shift referred to $I_{\mathrm{dc}}=0$ for samples (a) No. 2 and (b) No. 4. Some of the magnetoconductance traces are displayed in Figs. 3 and 4. The data were evaluated in a magnetic-field range from -30 to $30 \mathrm{mT}$. The dashed line is a guide to the eye. 
sample in two-wire configuration has to be symmetric upon the reversal of $B$. This restricts the phase $\varphi$ in Eq. (1) to either 0 or $\pi$, whereas there is no constraint for a four-wire configuration.

In a recent experiment with a metal ring interrupted by two tunnel junctions, the existence of the magnetoelectric $\mathrm{AB}$ effect was demonstrated. ${ }^{18}$ In this experiment, a voltage difference $V$ between the two arms of the ring induced an electrostatic phase shift $\delta \varphi=e V \tau_{d} / \hbar$, where $\tau_{d}$ is the diffusion time through the sample. ${ }^{9}$ This leads to a phase shift of the magneto-conductance oscillations as a function of $V$. The measured voltage difference necessary to induce a phase shift of $\pi$, was constant over the whole voltage range, since in those samples the two arms of the ring are on well-defined potentials as the voltage drop occurs across the tunnel junctions.

In contrast, in our experiment, the voltage drop occurs along the whole length of each arm of the ring. Therefore, the potential along the diffusive paths is not well defined, and we cannot expect a periodic phase shift as a function of $I_{\mathrm{dc}}$. Indeed we found a broad distribution of length of the plateaux with the same phase between 0.4 and $2.4 \mu \mathrm{A}$, when we evaluated a series of 129 magnetoconductance sweeps for different $I_{\mathrm{dc}}$ up to $\approx 50 \mu \mathrm{A}$ for sample No. 3 . With our sample parameters, the correlation energy is $E_{c, \mathrm{AB}}$ $\approx 22 \mu \mathrm{eV}$. With a ring resistance of $38 \Omega$, this corresponds to a current scale of approximately $0.6 \mu \mathrm{A}$, in reasonable agreement with the observed distribution of plateau lengths.

From a simple point of view, the $\mathrm{AB}$ oscillations arise from the interference of an electron that splits into two partial waves at one side of the ring. These waves traverse the ring in opposite arms and the amplitudes recombine at the other side. The interference is determined by the phase difference of the wave functions. From this simple argument, the phase of the wave functions should be the same at $B$ $=0$, provided that the arms have equal lengths. Hence at $B$ $=0$, the interference should be constructive and the conductance has a maximum.

However, the phase of the electronic wave function also depends on the configuration of the scattering centers. For this reason, the phase will usually not be the same in both arms of the ring, so that on the one hand, at $B=0$, an arbitrary interference between the partial waves might be possible. Indeed it was demonstrated that the $A B$ oscillations average to zero when the measurements are taken on a series of rings, ${ }^{13}$ each of them in a two-wire configuration with short interconnecting wires. On the other hand, the OR requires that for a ring in a two-wire configuration, the conductance at $B=0$ has either a maximum or a minimum. This means that the interference has to be either fully constructive or destructive.

The key to the resolution of these seemingly contradictory statements is that the above argument, considering only two wave functions, is too simple. For the correct calculation of the conductance, all transmitted and reflected partial waves have to be taken into account. ${ }^{3}$ They are not independent of each other but the OR impose correlations between them.

In order to visualize these correlations, the phase and the amplitude of the oscillations are displayed in a common

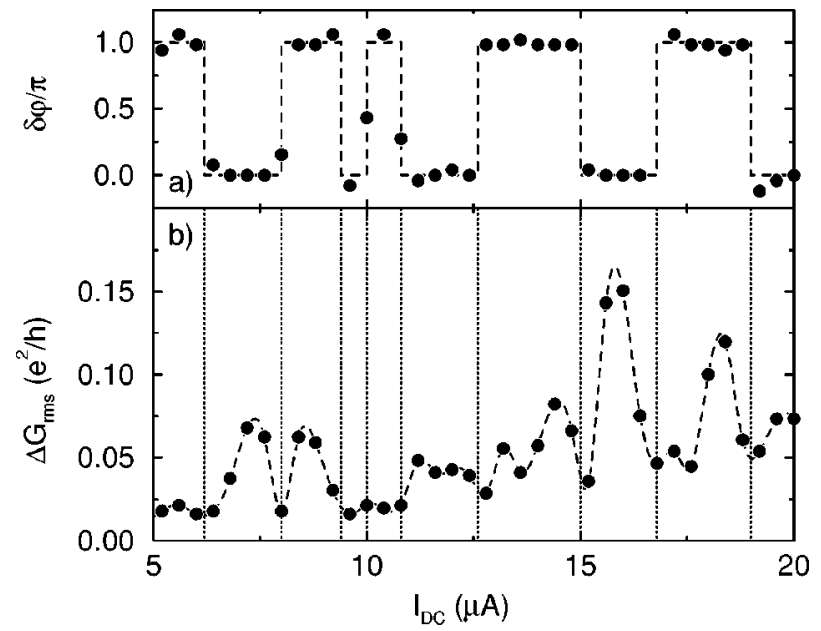

FIG. 7. (a) Phase shift referred to $I_{\mathrm{dc}}=7.6 \mu \mathrm{A}$ and (b) rms amplitude of the conductance oscillations vs $I_{\mathrm{dc}}$. The data were measured at sample No. 3 and evaluated in a magnetic-field range from -30 to $30 \mathrm{mT}$. The dashed lines are guides to the eye. The dotted-vertical lines mark the positions of a phase flip by $\pm \pi$.

graph in Fig. 7. The data were extracted from a series of MC traces measured on sample No. 3. It can be seen that both quantities are a function of $I_{\mathrm{dc}}$. There is a definite correlation between the variation of the amplitude and the phase; at each phase flip by $\pi$ there is a minimum of the oscillation amplitude. Hence no abrupt flip of the conductance oscillations occurs, but every variation of the phase by $\pm \pi$ is accompanied by a continuous decrease and subsequent increase of the oscillation amplitude.

Superimposed on the strong fluctuations of the oscillation amplitude, an overall increase of $\Delta G_{r m s}$ is observed. Averaging over a larger field interval than just [-30 mT,30 mT] yields $\Delta G_{r m s} \sim \sqrt{I_{\mathrm{dc}}}$, in agreement with the prediction for the conductance fluctuations in singly connected mesoscopic samples. ${ }^{14,19}$

Furthermore, the correlation between the partial waves evoked by the OR, results in a slight modulation of the local period, as, e.g., visible in Figs. 3 and 4: The arrows indicate the positions of conductance maxima for negative and positive effective magnetic field. In the two-wire sample (Fig. 3) the arrows shift towards $B=0$ for certain ranges of $I_{\mathrm{dc}}$ or they separate from each other. This means that the oscillations are compressed or expanded in those current ranges. The arrows in Fig. 4 move simultaneously to the left or to the right, keeping the oscillation period constant.

In a recent experiment on semiconductor rings in twowire configuration with a few electron transmission channels, the phase of the oscillations could be modified by variation of a gate voltage. ${ }^{5}$ A phase flip by $\pi$ of the fundamental oscillation was accompanied by the occurrence of a dominating higher harmonic with $h / 2 e$ periodicity. In our experiment on diffusive rings with many electron transmission channels, there are so many degrees of freedom for the electronic partial waves that they arrange to a minimum of the oscillation amplitude. However, the center of the Fourier peak seems to shift to higher $B_{p e r}$ values when the amplitude approaches a minimum. A naive interpretation is that the partial waves 

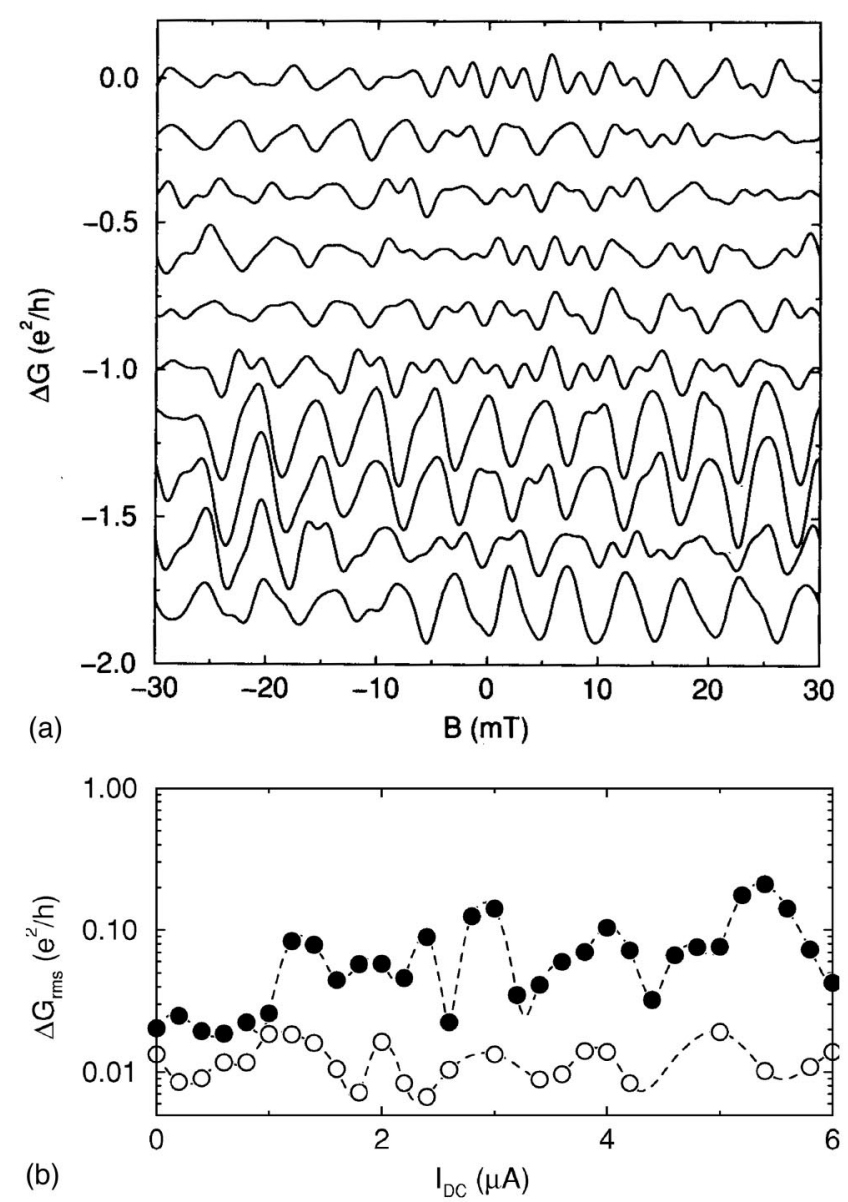

FIG. 8. (a) Magneto conductance at $T=90 \mathrm{mK}$ of sample No. 1 for $I_{\mathrm{dc}}=0,0.2,0.4, \ldots, 1.8 \mu \mathrm{A}$. (b) Amplitudes of the $h / e$ (closed symbols) and $h / 2 e$ (open symbols) oscillations. The data are evaluated in a magnetic-field range from -30 to $30 \mathrm{mT}$. The dashed lines are guides to the eye.

traveling along the inner perimeter of the ring have higher weight at those $I_{\mathrm{dc}}$ values. In summary, correlations between the partial waves within one ring arm (giving rise to the minimum of the oscillation amplitude whenever a phase flip is necessary) as well as between the two ring arms, are necessary to fulfill the OR.

Finally, let us briefly discuss how strict the given criterion is for the definition of two-wire and four-wire configuration. We investigated sample No. 1, where the separation of the connecting wires into current and voltage leads was not directly at the ring but at $500 \mathrm{~nm}<L_{\varphi}$ from the ring, i.e., within the phase-coherent volume, as required for a fourwire configuration. Figure 8 displays the MC at low fields for various values of $I_{\mathrm{dc}}$. For this sample we observe not only $h / e$ but also $h / 2 e$ oscillations, due to the much higher $L_{\varphi} \cdot{ }^{21}$ Both amplitudes are modulated by $I_{\mathrm{dc}}$, but no clear correlation between the modulation was observed [see Fig. 8(b)]. The behavior of the phase shifts displayed in Fig. 9 is interesting; although the $\mathrm{MC}$ is not symmetric around the offset

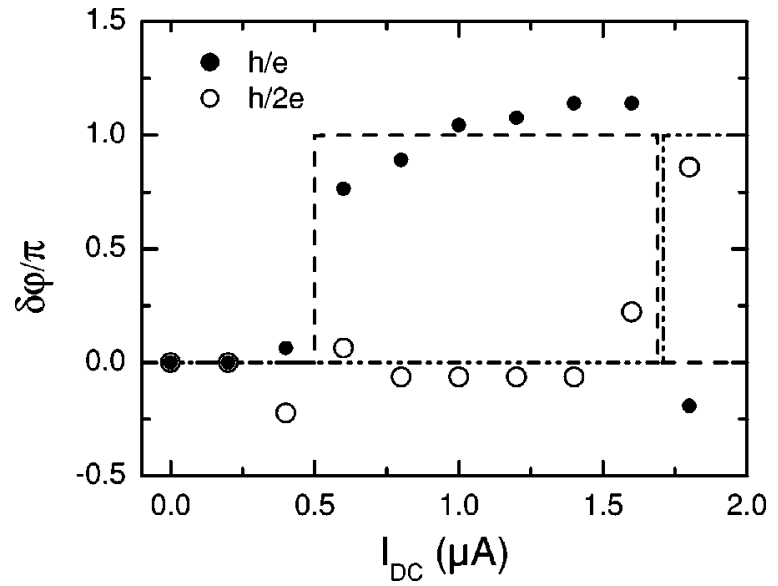

FIG. 9. Phase shifts of sample No. 1 referred to $I_{\mathrm{dc}}=0$ of the magneto-conductance traces displayed in Fig. 8. The data were evaluated in a magnetic-field range from -100 to $100 \mathrm{mT}$. The dashed and dash-dotted lines are guides to the eye.

value of the magnetic field $B=4.5 \mathrm{mT}$, the phase does not shift continuously but jumps between $\varphi=0$ and $\pi$ for both the $h / e$ and the $h / 2 e$ oscillations. Also the UCF's are asymmetric (not shown). This observation is in agreement with the findings of Umbach et al. ${ }^{13}$ who always observed for a four-wire configured series of rings (each of the rings was in two-wire configuration with short interconnecting leads) phases that were either 0 or $\pi$.

In conclusion, although the microscopic phase of the electronic wave function is varied continuously by a modification of $I_{\mathrm{dc}}$, the macroscopic phase of the conductance oscillations varies in a quantized manner for two-wire configurations. However, there is no abrupt change in the MC. Rather, the macroscopic phase flip is accommodated by a rearrangement of the individual electron phases to produce an interference pattern leading to a minimum of the oscillation amplitude. This continuous variation of the interference on the microscopic level is also visible (cf. Fig. 3) as a slight shift of the oscillation frequency whenever a phase flip occurs. Thus, the suppression at macroscopic phase flips indicates the rearrangement at the microscopic level. This confirms the notion that the current induced variation of the energy of the electrons leads to an altered microscopic interference pattern. In the case of a four-wire configuration, this variation of the interference does not need to be correlated in both arms of the ring, as can be seen in the arbitrary values of the phase shift of the oscillations.

\section{ACKNOWLEDGMENTS}

The authors thank D. Gerthsen, Laboratorium für Elektronenmikroskopie, for lending an electron microscope for e-beam lithography and P. Pfundstein for operation of the microscope. This work was supported by the Deutsche Forschungsgemeinschaft through SFB 195. 
*Present address: OCMD, Agilent Technologies, Herrenberger Str. 130, D-71034 Böblingen, Germany.

†Corresponding author. Email address: elke.scheer@unikonstanz.de

${ }^{1}$ L. Onsager, Phys. Rev. 38, 2265 (1931).

${ }^{2}$ H.B.G. Casimir, Rev. Mod. Phys. 17, 343 (1945).

${ }^{3}$ M. Büttiker, Phys. Rev. Lett. 57, 1761 (1986).

${ }^{4}$ A. Yacoby, R. Schuster, and M. Heiblum, Phys. Rev. B 53, 9583 (1996).

${ }^{5}$ S. Pedersen, A.E. Hansen, A. Kristensen, C.B. Sørensen, and P.E. Lindelof, Phys. Rev. B 61, 5457 (2000).

${ }^{6}$ A.D. Benoit, S. Washburn, C.P. Umbach, R.B. Laibowitz, and R.A. Webb, Phys. Rev. Lett. 57, 1765 (1986).

${ }^{7}$ R.A. Webb, S. Washburn, C.P. Umbach, and R.B. Laibowitz, Phys. Rev. Lett. 54, 2696 (1985).

${ }^{8}$ A.D. Stone and Y. Imry, Phys. Rev. Lett. 56, 189 (1986).

${ }^{9}$ Y. Aharonov and D. Bohm, Phys. Rev. 115, 485 (1959).

${ }^{10}$ A. Müller-Groeling, Phys. Rev. B 47, 6480 (1993).

${ }^{11}$ D.P. DiVincenzo and C.L. Kane, Phys. Rev. B 38, 3006 (1988).

${ }^{12}$ For the $\mathrm{AB}$ oscillations in twofold connected structures made of quasi-one-dimensional branches $\left(w<L_{\varphi}, L\right)$, the phasecoherence length does not occur in $B_{c}$, even when it is smaller than the sample length $L$, because only those electronic partial waves contribute to the $\mathrm{AB}$ interference pattern, which have transversed the whole sample phase coherently. However, $L_{\varphi}$ is important for the energy and field scales appearing in the universal conductance fluctuations due to diffusive motion and interference within the ring branches (see, e.g., Refs. 10 and 11).
${ }^{13}$ C.P. Umbach, C. van Haesendonck, R.B. Laibowitz, S. Washburn, and R.A. Webb, Phys. Rev. Lett. 56, 386 (1986).

${ }^{14}$ R. Häussler, H.B. Weber, and H.v. Löhneysen, J. Low Temp. Phys. 118, 467 (2000).

${ }^{15}$ It is unclear why this particular sample had a much higher $L_{\varphi}$ than the others.

${ }^{16}$ A.G. Aronov and Yu.V. Sharvin, Rev. Mod. Phys. 59, 755 (1987).

${ }^{17}$ C. Terrier, D. Babić, C. Strunk, T. Nussbaumer, and C. Schönenberger, cond-mat/0103486 (unpublished).

${ }^{18}$ A.v. Oudenaarden, M.H. Devoret, Y.V. Nazarov, and J.E. Mooij, Nature (London) 391, 768 (1998).

${ }^{19}$ This dependence is valid for a current range $I_{c}<I_{\mathrm{dc}}<I_{\text {inel }}$ and temperatures $k_{B} T \ll e R I_{\mathrm{dc}}$, where $I_{c}=E_{c} / e R$ is the current related to the Touless energy and $I_{\text {inel }}$ a current range above which averaging due to inelastic scattering sets in (see Refs. 17 and 20).

${ }^{20}$ A.I. Larkin and D.E. Khmel'nitskii, Zh. Éksp. Teor. Fiz. 91, 1815 (1986) [Sov. Phys. JETP 64, 1075 (1986)].

${ }^{21}$ There are two possible origins of $h / 2 e$ oscillations: The first one would be weak localization, which gives rise to the so-called Altshuler-Aronov-Spivak effect in twofold connected geometries. These oscillations, however, are suppressed by larger absolute values of the magnetic field. The second possibility would be the first harmonic of the $\mathrm{AB}$ oscillations, i.e., electron partial waves, which travel twice around the ring. The latter are not smeared out by large magnetic fields. Since the oscillations observed here persist up to the highest measuring fields, we attribute them to $\mathrm{AB}$ oscillations. 